\title{
Affiliation aux pairs déviants et désengagement scolaire chez les adolescents : la participation aux activités parascolaires peut-elle agir comme facteur de protection? Peer deviance and student disengagement in adolescence: Does participation in extracurricular activities can act as a protective factor?
}

\author{
Marie-Michèle Brossard, Isabelle Archambault et Stéphane Cantin
}

Volume 43, numéro 2, 2014

URI : https://id.erudit.org/iderudit/1061185ar

DOI : https://doi.org/10.7202/1061185ar

Aller au sommaire du numéro

Éditeur(s)

Revue de Psychoéducation

ISSN

1713-1782 (imprimé)

2371-6053 (numérique)

Découvrir la revue

Citer cet article

Brossard, M.-M., Archambault, I. \& Cantin, S. (2014). Affiliation aux pairs déviants et désengagement scolaire chez les adolescents : la participation aux activités parascolaires peut-elle agir comme facteur de protection ? Revue de psychoéducation, 43(2), 273-298. https://doi.org/10.7202/1061185ar

\section{Résumé de l'article}

De nombreux jeunes suivent une trajectoire de désengagement scolaire qui ultimement, se traduit par l'abandon des études sans diplôme ni qualifications. Parmi les prédicteurs susceptibles de contribuer à cette trajectoire négative, l'affiliation à des pairs déviants en début d'adolescence est un facteur important à considérer. Par contre, d'autres contextes extrafamiliaux comme le contexte des activités parascolaires pourraient également jouer un rôle dans cette dynamique et compenser, voire protéger les élèves de l'influence négative de leurs pairs. Sur cette base, la présente étude cherche à étudier le rôle modérateur de la participation aux activités parascolaires pour rendre compte du lien entre l'affiliation aux pairs déviants et le désengagement scolaire des adolescents. L'échantillon est composé de 448 adolescents du secondaire fréquentant trois écoles de milieu défavorisé de la région montréalaise. Les résultats indiquent qu'au-delà de facteurs de risque reconnus, la participation aux activités parascolaires peut agir à la fois comme facteur de risque et comme facteur de protection chez les filles qui ont des amis déviants, mais pas chez les garçons. Les implications de ces résultats pour la recherche et l'intervention psychoéducative sont abordées en discussion.
Tous droits réservés ( $\odot$ La Corporation de la Revue Canadienne de Psycho-Éducation, 2014
Ce document est protégé par la loi sur le droit d'auteur. L'utilisation des services d'Érudit (y compris la reproduction) est assujettie à sa politique d'utilisation que vous pouvez consulter en ligne. 


\section{Affiliation aux pairs déviants et désengagement scolaire chez les adolescents : la participation aux activités parascolaires peut-elle agir comme facteur de protection?}

\section{Peer deviance and student disengagement in adolescence: Does participation in extracurricular activities can act as a protective factor?}

\section{M.-M. Brossard ${ }^{1}$ \\ I. Archambault ${ }^{1}$ \\ S. Cantin ${ }^{1}$}

École de psychoéducation, Université de Montréal

\section{Correspondance :}

Isabelle Archambault

École de psychoéducation,

Université de Montréal,

Montréal, Qc, C.P.6128,

succ. Centre-Ville, H3C 3J7

isabelle.archambault@umontreal.ca

\section{Résumé}

De nombreux jeunes suivent une trajectoire de désengagement scolaire qui ultimement, se traduit par l'abandon des études sans diplôme ni qualifications. Parmi les prédicteurs susceptibles de contribuer à cette trajectoire négative, l'affiliation à des pairs déviants en début d'adolescence est un facteur important à considérer. Par contre, d'autres contextes extrafamiliaux comme le contexte des activités parascolaires pourraient également jouer un rôle dans cette dynamique et compenser, voire protéger les élèves de l'influence négative de leurs pairs. Sur cette base, la présente étude cherche à étudier le rôle modérateur de la participation aux activités parascolaires pour rendre compte du lien entre l'affiliation aux pairs déviants et le désengagement scolaire des adolescents. L'échantillon est composé de 448 adolescents du secondaire fréquentant trois écoles de milieu défavorisé de la région montréalaise. Les résultats indiquent qu'au-delà de facteurs de risque reconnus, la participation aux activités parascolaires peut agir à la fois comme facteur de risque et comme facteur de protection chez les filles qui ont des amis déviants, mais pas chez les garçons. Les implications de ces résultats pour la recherche et l'intervention psychoéducative sont abordées en discussion.

Mots-clés: Désengagement scolaire, activités parascolaire, déviance des pairs.

\section{Abstract}

Many youngsters follow a downward trajectory of school disengagement which eventually leads to school dropout. Among predictors that contribute to this negative trajectory, deviant peer affiliation is an important factor to consider. Thus, because adolescents have multiples sources of influence, other extrafamilial contexts such as the context of extracurricular activities could also influence student disengagement in school. It could compensate and even protect students from the negative influence of their peers. As such, 


\section{4}

the present research aims to study the moderating role of extracurricular activity participation on the relationship between deviant peers' affiliation and adolescent disengagement in school. The study sample comprised 448 adolescents from three low-income secondary schools in the Montreal area. Results show that beyond important risk factors, student participation in extracurricular activities act a protective factor for girls, but not for boys. The implications of our results in terms of research and intervention are discussed.

\section{Keywords: School disengagement, extracurricular activities, peer deviancy.}

Le décrochage scolaire est devenu une préoccupation majeure dans les sociétés occidentales. En abandonnant l'école avant l'obtention d'un diplôme, les jeunes sont aux prises avec des limites sur le marché de l'emploi les rendant vulnérables à une plus grande dépendance sociale, un taux de chômage plus élevé et une moins bonne qualité de vie à l'âge adulte (Dubow, Huesmann, Boxer, Pulkkinen, \& Kokko, 2006; Mortimer, Vuolo, Staff, Wakefield, \& Xie, 2008; Rumberger, 1987; Wiesner, Vondracek, Capaldi, \& Porfeli, 2003). Or, la majorité des jeunes ne deviennent pas décrocheur du jour au lendemain. De nombreux décrocheurs suivent une trajectoire graduelle de désengagement scolaire qui ultimement, se traduit par l'abandon des études sans diplôme, ni qualifications (Janosz, Archambault, Morizot, \& Pagani, 2009a). Parmi les facteurs susceptibles de contribuer à cette trajectoire négative, l'affiliation à des pairs déviants en début d'adolescence suscite un intérêt considérable dans les écrits scientifiques (Dodge, Lansford, \& Dishion, 2006; Stanard, Belgrave, Corneille, Wilson, \& Owens, 2010). En effet, plusieurs auteurs suggèrent que la fréquentation de pairs aux comportements délinquants contribue au désengagement et à l'échec scolaire des élèves (Newcomb, Abbott, Catalano, Hawkins, Battin-Pearson, \& Hill, 2002; Hirschfield \& Gasper, 2011; Stanard et al., 2010). Par ailleurs, comme le début de l'adolescence est une période critique d'exploration où les jeunes sont sujets à de multiples influences (Damon \& Hart, 1988), il demeure plausible que d'autres contextes extrafamiliaux, notamment la participation à des activités parascolaires, puissent également compenser voire même protéger les élèves de l'influence négative de leurs pairs. Cette étude vise spécifiquement à vérifier cette hypothèse en examinant le rôle modérateur de la participation aux activités parascolaires pour rendre compte de l'association entre l'affiliation aux pairs déviants et le désengagement scolaire des adolescents.

\section{L'engagement scolaire}

Le rôle déterminant de l'engagement scolaire sur la réussite académique des adolescents n'est certes plus à démontrer. L'idée que l'engagement scolaire consiste en un processus qui diminue au fil des années et évolue sur un continuum allant d'un pôle positif à un pôle négatif fait également consensus (Archambault, Janosz, Fallu, \& Pagani, 2009b; Dotterer, McHale, \& Crouter, 2007). Par contre, cette notion est trop souvent conçue de multiples façons dans les écrits scientifiques. Afin de regrouper ses multiples conceptions, Fredrick, Blumenfeld et Paris (2004) proposent une définition exhaustive selon laquelle l'engagement scolaire comprendrait trois dimensions, soit l'engagement comportemental, cognitif et affectif. Selon ces auteurs, l'engagement comportemental réfère au degré de conformité de l'élève aux consignes de la classe ainsi qu'à son implication dans les activités et les discussions proposées. Cette dimension inclut la présence en 
classe, l'adoption de conduites disciplinées, le respect des règles et des consignes. La composante cognitive concerne davantage la motivation de l'élève à persévérer en classe ainsi que ses habiletés d'autorégulation et d'organisation. Finalement la sphère affective fait référence aux sentiments, aux intérêts et aux perceptions du jeune face à l'école. Elle réfère également au sentiment d'appartenance de l'adolescent et à son enthousiasme à l'idée d'apprendre de nouvelles choses.

Dans l'ensemble, ces trois dimensions qui peuvent être conçues tant positivement que négativement sont associées au succès et à la persévérance scolaire des élèves (Connell, 1990; Furrer, Skinner, Marchand, \& Kindermann, 2006; Zepke \& Leach, 2010). En effet, les élèves dits engagés sur l'une ou l'autre de ces dimensions sont plus susceptibles de vivre une expérience scolaire positive et de réussir alors qu'à l'inverse, les élèves désengagés présentent plus de risques de comportements perturbateurs, de grandes difficultés académiques et de décrochage scolaire (Appleton, Christenson, Dongjin, \& Reschly, 2006; Archambault et al., 2009b). II existerait par ailleurs des différences notables entre les garçons et les filles et ce, pour chacune des dimensions (Lam et al., 2012; Warrington, Younger, \& Williams, 2000). Plus spécifiquement, les garçons seraient moins intéressés par l'école et par les activités d'apprentissage que les filles, passeraient moins de temps à faire leurs devoirs et seraient moins enthousiastes à l'idée de poursuivre leurs études (Barber, 1996; Cox, 2000; Hatzichristou \& Hopf, 1996). Ils auraient également moins tendance à planifier, à s'organiser et à s'autoréguler dans leurs activités scolaires (Ablard \& Lipschultz, 1998; Kenney-Benson, Pomerantz, Ryan, \& Patrick, 2006; Zimmerman \& Martinez-Pons, 1990) alors qu'à l'inverse, les filles participeraient davantage aux activités proposées (Sirin, Rogers-Sirin, \& Collins, 2010). Malgré ces différences, l'engagement comportemental, c'est-à-dire l'adoption de comportements disciplinés, le respect des consignes, ainsi que la présence et l'implication dans les activités de classe, demeure le meilleur prédicteur proximal de la réussite scolaire, et ce, chez les garçons comme chez les filles (Archambault et al., 2009a; Finn \& Cox, 1992; Finn \& Rock, 1997; Strambler \& Weinstein, 2010). En raison du rôle central de cette dimension dans la trajectoire associée au décrochage scolaire (Archambault et al., 2009b), la présente étude s'intéressera spécifiquement à l'influence des pairs déviants et de la participation aux activités parascolaires sur le désengagement comportemental des adolescents et adolescentes.

\section{L'affiliation à des pairs déviants comme prédicteur du désengagement scolaire}

La gravité et l'intensité des comportements déviants chez les jeunes varient selon la trajectoire de conduite délinquante de chacun (Moffitt, 1993). Un consensus semble toutefois établi sur le fait qu'un acte déviant a lieu à partir du moment où il y a transgression d'une norme établie (Becker, 1985; Cusson, 1992; Mucchielli, 1999). Cette définition très large peut inclure tant les comportements d'impolitesse, le flânage et la consommation de psychotropes que l'introduction par infraction, l'engagement dans un combat ou le port d'arme à l'école. Le point commun de tous ces comportements réside dans le fait qu'ils sont condamnés par différentes normes sociales. Ainsi, l'acte déviant fait référence à toute conduite qui est socialement désapprouvée, autant formellement (par la loi) qu'informellement (par les règles et les normes sociales) (Lanctôt, 2006). 
Selon Moffit (1993), la déviance à l'adolescence est souvent un phénomène normatif. Par contre, il existe d'importantes différences individuelles, certains jeunes étant plus à risque que d'autres de suivre des trajectoires déviantes ancrées et problématiques. C'est notamment le cas des garçons chez qui la déviance s'exprimerait davantage par des comportements d'agressions physiques, des crimes contre les personnes et les biens que chez les filles (Lanctôt, 2005). En effet, bien que les filles et les garçons soient aussi susceptibles de commettre des vols ou de consommer des psychotropes (Moretti, Odgers, \& Jackson, 2004), le phénomène de bandes de filles reste rare et $95 \%$ des actes de violence graves sont commis par des garçons (Roché, 2001). À l'inverse, la délinquance des filles s'exprimerait davantage à partir de délits mineurs, de comportements d'agressivité relationnelle et de violence dirigée vers soi-même (Moretti et al., 2004; Verlaan \& Déry, 2006).

Par ailleurs, au-delà des différences sexuelles, il existe un consensus dans la littérature quant à l'influence criminogène des pairs, l'affiliation à des pairs déviants serait ainsi positivement associée au développement des conduites déviantes à l'adolescence (Burk, Steglich, \& Snijders, 2007; Morizot \& Leblanc, 2000; Osgood, Wilson, O'Malley, Bachmman, \& Johnson, 1996). Ainsi, des études ont démontré que la socialisation avec des pairs déviants dès le début de l'adolescence aurait un impact négatif sur l'engagement et la réussite scolaire (Newcomb et al., 2002; Hirschfield \& Gasper, 2011; Stanard et al., 2010). À l'inverse, les adolescents dont les pairs sont investis et performants à l'école sont plus engagés et réussissent mieux (Mounts \& Steinberg, 1995; Veronneau \& Dishion, 2011). Dans ce sens, une étude longitudinale de Kindermann (2007) conclut que l'engagement des amis à l'automne prédirait l'engagement de l'adolescent au printemps de la même année scolaire. De la même façon, Burk, Kerr et Stattin (2008) démontrent que le désengagement scolaire des amis influencerait la déviance à l'adolescence qui, en retour, serait associée à une diminution de l'engagement.

Cette influence réciproque des amis sur le désengagement peut notamment être expliquée par le processus de socialisation qui prend ses racines dans la théorie de l'apprentissage social de Bandura (1986). Selon cette théorie, l'individu apprendrait par l'observation et l'imitation de comportements qui font l'objet de récompenses et de réussites, en l'occurrence, par renforcement différentiel (Bandura, 1986). II aurait donc tendance à modeler les comportements d'autres individus auxquels il est attaché et peut s'identifier, surtout quand ces comportements semblent associés à des gains. Dans ce sens, les travaux de Dishion et Patterson (1997) démontrent que les délinquants renforcent positivement les discussions et les comportements antisociaux de leurs amis alors qu'ils ignorent les conduites et les attitudes dites prosociales. Les pairs deviennent ainsi des acteurs clés dans le développement de la déviance dans la mesure où ils représentent un public fidèle pour renforcer les conduites transgressant les normes établies (Osgood et al., 1996). Puisque la manifestation de comportements antisociaux à l'école est associée à la manifestation de ces mêmes comportements à l'extérieur de l'école, les adolescents qui développent des affinités avec des pairs antisociaux sont plus susceptibles de modeler ces comportements dans différents contextes, notamment celui de la classe. Par ailleurs, selon le même processus, les amis peuvent aussi devenir des modèles positifs. En effet, plusieurs auteurs démontrent que les jeunes 
sont plus susceptibles de s'engager à l'école s'ils s'affilient avec des amis qui sont eux-mêmes engagés et s'ils reçoivent du renforcement positif de la part de ces derniers (Ryan \& Patrick, 2001; Sage \& Kindermann, 1999).

L'influence des pairs déviants sur le désengagement de l'élève peut également être expliquée par un processus de cohésion (Cohen, 1958; Lott, 1961). La cohésion réfère à l'idée que les adolescents tissent des liens très forts avec leurs amis et qu'ils sont très solidaires entre eux. La force de ces relations interpersonnelles s'évalue par le temps passé avec les pairs, notamment en terme de durée et de fréquence, par l'ampleur des discussions et par la confiance mutuelle qui s'établit entre eux (Cook, Herman, Phillips, \& Settersen, 2002; Hartup, 1996; Pellegrini \& Blatchford, 2000). La nature et l'ampleur de la cohésion que développent les garçons et les filles avec leurs amis ne seraient toutefois pas nécessairement similaires. Selon la théorie de la différence des genres, les filles auraient plus tendance à investir dans leurs relations d'amitié (Gorman-Smith, 2003; Kiuru, Nurmi, Aunola, Salmela, \& Aro, 2009), seraient plus attachées à leurs amies que les garçons et seraient plus sensibles aux opinions et à la pression exercée par leurs pairs (Benenson et Schinazi, 2004), ce qui suggère qu'elles seraient plus influençables. Elles se laisseraient également entraîner davantage par leurs amies à commettre des vols (McCarthy, Felmlee, \& Hagan, 2004), à avoir des comportements antisociaux (Mahoney \& Stattin, 2004), ainsi que dans leur permissivité sexuelle (Gorman-Smith, 2003). Finalement, comme l'affiliation à des pairs déviants semble être anxiogène et souvent conflictuelle (Marcus, 1996), les filles souffriraient davantage que les garçons de cette association et seraient plus angoissées lors des conflits amicaux (Turner \& Avison, 2003). À l'opposé, les garçons étant plus compétitifs et indépendants entre amis, ils seraient moins susceptibles de se laisser influencer.

\section{Lien entre la participation à une activité parascolaire et l'engagement scolaire}

Dans les écrits scientifiques, le concept d'activité parascolaire est défini et opérationnalisé de multiples façons. Certains auteurs parlent d'activités structurées (par exemple, participation à une équipe sportive) ou non structurées (par exemples, fréquentation d'une maison de jeunes) (Covay \& Carbonaro, 2010), alors que d'autres mentionnent l'aspect volontaire de la participation (Larson, 2000). Dans le même sens, certains travaux abordent uniquement les activités parascolaires de nature sportive (Gardner, Roth, \& Brooks-Gunn, 2009), alors que d'autres incluent les arts et le bénévolat (Eccles, Barber, Stone, \& Hunt, 2003). Face à cette divergence de vocabulaire, Larson (2000) tente une définition plus complète et exhaustive en décrivant l'activité parascolaire comme : " toute activité structurée à laquelle un élève participe volontairement à l'extérieur du cadre académique, tel que les sports d'équipe, les sports individuels, les arts, le bénévolat où il y a interaction avec un adulte compétent et des pairs, et où l'objectif est de développer des habiletés progressives dans un domaine précis » (p.174).

La majorité des travaux sur le sujet documente les effets bénéfiques de la participation aux activités parascolaires sur l'engagement scolaire et le développement global des adolescents (Dottorer et al., 2006; Feldman \& Matjasko, 


\section{8}

2005; Fredricks \& Eccles, 2008; Newmann, 1992). Dans son modèle bioécologique, Bronfenbrenner (1979) mentionne notamment que le développement d'un individu résulte des interactions continuelles et réciproques entre l'organisme et son environnement. Selon cette perspective, le fait d'être exposé à plusieurs types de milieux tels que le cadre parascolaire aurait des effets bénéfiques considérables sur le développement de l'individu. Plusieurs auteurs ont confirmé ce modèle en affirmant que l'implication dans des activités parascolaires (mesuré par le nombre d'activités ainsi que le temps passé dans chacune des activités) prédit le développement positif à l'âge adulte en favorisant de plus grandes aspirations éducationnelles, une meilleure qualité de travail à 25 ans, une participation active au processus politique, une plus grande implication dans les activités de bénévolat ainsi qu'une meilleure santé mentale (Barber, Eccles, \& Stone, 2001; Marsh, 1992; Youniss, McLellan, Su, \& Yates, 1999). Les contextes au sein desquels se déroulent les activités parascolaires seraient également propices à l'exécution de plusieurs tâches développementales telles que la socialisation, la formation d'identité et la connaissance de soi (Dotterer et al., 2006; Eccles \& Templeton, 2002; Feldman \& Matjasko, 2005).

Par ailleurs, les paramètres considérés lorsqu'il s'agit d'évaluer la participation aux activités parascolaires varient largement, ce qui influence les résultats obtenus d'une étude à l'autre. Quatre dimensions reviennent toutefois plus fréquemment dans les écrits scientifiques. Premièrement, de nombreuses études s'attardent à la diversité des activités, en l'occurrence, le nombre d'activités auxquelles participent les jeunes. Par exemple, Barber et Eccles (1997) rapportent que la diversité des activités parascolaires prédirait l'engagement scolaire des adolescents, leurs résultats académiques, ainsi qu'un usage moindre de marijuana chez les élèves de 12e année. Deuxièmement, le type d'activité dans laquelle l'élève choisit de s'investir aurait également des effets sur son développement. Ainsi, des études suggèrent que certaines activités telles que les activités religieuses et le bénévolat favoriseraient l'affiliation à des pairs prosociaux alors que d'autres, tels que les sports d'équipe, favoriseraient l'affiliation à des pairs plus déviants, et ce, indépendamment du sexe et du statut socioéconomique de l'élève (Eccles \& Barber, 1999; Marsh \& Kleitman, 2002; McHale, Crouter, \& Tucker, 2001). La troisième dimension concerne la durée de l'activité. Plusieurs auteurs démontrent que les jeunes qui sont impliqués depuis plus longtemps au sein d'une activité parascolaire font preuve d'une plus grande résilience psychosociale, d'une meilleure réussite académique et possèdent un réseau social plus positif (Darling, 2005; Eccles et al., 2003). Enfin, la dernière dimension utilisée dans la littérature consiste en l'intensité des activités parascolaires, soit le nombre d'heures hebdomadaires qu'y passe l'élève. Puisqu'il s'agit de l'une des mesures les plus communes pour évaluer la participation aux activités parascolaires, la présente étude sera basée sur cette dimension. De façon générale, des chercheurs ont trouvé une corrélation positive entre le nombre d'heures consacrées à une activité par semaine et l'augmentation de l'engagement affectif et de l'intérêt envers l'école (Eccles \& Fredricks, 2006; Marsh \& Kleitman, 2002).

Malgré la multitude d'études démontrant la contribution bénéfique de la participation aux activités parascolaires sur l'engagement scolaire des adolescents, certaines autres études arrivent à des résultats contraires, démontrant que la 
participation aux activités parascolaires peut avoir des effets néfastes sur le développement. Selon le modèle de Coleman (1961), les activités non académiques nuiraient à la réussite scolaire des élèves en accaparant du temps qui aurait pu être consacré aux études. Ainsi, les activités parascolaires interfèrent parfois avec l'investissement de l'élève dans ses travaux académiques. Également, certains chercheurs ont démontré que les jeunes sportifs sont généralement perçus comme plus populaires et, par conséquent, reconnus comme moins conformistes et plus à risque de comportements déviants (Cillessen, 2011; Fallu, Brière, Vitaro, Cantin, \& Borge, 2011; Vaillancourt \& Hymel, 2006). Dans ce sens, certains types d'activités tels que les sports d'équipes seraient reliés positivement à une augmentation de la consommation d'alcool et de drogues (Denault \& Poulin, 2011; Eccles et al., 2003; Fauth, Roth, \& Brooks-Gunn, 2007; Lansford, 2006). Finalement, d'autres travaux suggèrent que des jeunes qui présentent des comportements déviants peuvent avoir une influence négative sur les comportements d'autres élèves qui participent aux mêmes activités parascolaires dans le cas où ces activités sont moins structurées et encadrées par des adultes (Mahoney \& Stattin, 2000; Stattin, Kerr, Mahoney, Persson, \& Magnusson, 2005).

\section{La participation aux activités parascolaires comme facteur de protection}

Parmi les différents processus qui pourraient permettre d'expliquer le lien entre l'affiliation aux pairs déviants et le désengagement comportemental, l'importance des contextes relationnels est inévitable. En effet, comme le démontre le modèle des périodes développementales de Damon et Hart (1988), l'adolescence est une période critique où les jeunes sont à la recherche d'une identité propre et cherchent à établir une indépendance familiale. La présence de contextes extrafamiliaux devient importante, et les premiers environnements permettant cette distance familiale sont l'école et le groupe de pairs. Considérant que la transition de l'école primaire au secondaire contraint les adolescents à composer avec une nouvelle réalité sociale complexe qui appelle à l'établissement de nouveaux réseaux sociaux (Cillessen \& Mayeux, 2007), les premières années à l'école secondaire sont d'autant plus importantes qu'elles correspondent au moment où les jeunes expérimentent différentes relations d'amitié afin de trouver ce qui leur convient.

De nombreuses études démontrent que la participation aux activités parascolaires peut servir de contexte relationnel favorisant l'affiliation à des pairs prosociaux (Agnew \& Peterson, 1989; Barber, Eccles, Stone, \& Hunt, 2005 2005; Burke et al., 2008), jouant ainsi un rôle modérateur dans la relation entre l'affiliation aux pairs déviants et le désengagement comportemental. Par l'entremise de différents mécanismes, le fait de s'investir dans une activité parascolaire dès l'entrée au secondaire pourrait ainsi protéger les élèves de l'influence négative de leurs pairs et ce, surtout pour les élèves qui présentent d'importantes difficultés académiques. En effet, le contexte où se déroule les activités parascolaires serait souvent le seul contexte positif l'intérieur de l'école permettant aux élèves à risque de pratiquer et de développer leurs habiletés, notamment leur capacité d'autodiscipline, d'écoute et d'organisation (Brown \& Theobald, 1998; Gilman, Meyers, \& Perez, 2004; Marsh, 1992). 
La similitude entre les adolescents et leurs amis est l'un de ces mécanismes. La similarité entre amis s'explique en partie par un processus de sélection mutuelle qui réfère à la tendance des individus à se choisir mutuellement sur la base de certaines caractéristiques communes telles que les valeurs, les intérêts et les comportements avant même le début de leur amitié (Haselager, Hartup, van Lieshout, \& Riksen-Walraven, 1998). Cette similarité des amis continue de croître une fois la relation formée, résultat de l'influence qu'ils exercent l'un sur l'autre. Or, il est probable que la plupart des adolescents qui s'inscrivent à une même activité parascolaire partagent des intérêts et des valeurs prosociales communes. Plusieurs auteurs ont confirmé cette hypothèse en concluant que la participation aux activités parascolaires, surtout lorsque ces activités sont organisées dans le contexte scolaire, favorise l'adhésion aux valeurs prosociales en exposant les adolescents à des modèles et des pairs prosociaux qui sont eux-mêmes moins déviants (Agnew \& Peterson, 1989; Mahoney, 2000; Osgood et al., 1996). Ainsi, même si le jeune fréquente des amis déviants au début du secondaire, le fait d'être exposé à des pairs prosociaux dans le cadre d'activités parascolaires se déroulant à l'école pourrait agir comme facteur de protection contre un éventuel désengagement.

Dans le même sens, d'autres travaux démontrent que l'implication dans une activité parascolaire diminue les opportunités d'actes déviants et de délinquance dans la mesure où l'adolescent n'a pas de temps libre pour s'y consacrer (Haynie \& Osgood, 2005). Ainsi, selon la théorie des activités routinières, les jeunes qui consacrent leur temps libre (généralement entre $16 \mathrm{~h}$ et $22 \mathrm{~h}$ ) à des activités non structurées telles que les flâneries dans les parcs et les arcades ont plus tendance à s'adonner à des actions déviantes avec des pairs délinquants (Agnew \& Petersen, 1989; Lanctôt \& Le Blanc, 1997; Zill, Nord, \& Loomis, 1995).

Par ailleurs, certaines études suggèrent à l'inverse que l'influence des activités parascolaires pourrait plutôt être négative, notamment lorsque les jeunes qui y participent présentent un profil plus problématique. En effet, sous l'influence de la socialisation, la participation à des activités parascolaires non structurées et non supervisées peut amener les adolescents à s'investir auprès d'un groupe déviant et adopter des comportements antisociaux (Agnew \& Petersen, 1989; Osgood et al., 1996; Mahoney, 2000). Ainsi, le fait d'être exposé à des pairs déviants dans le cadre d'activités parascolaires pourrait agir comme facteur aggravant et promouvoir le désengagement.

\section{Objectifs et hypothèses de recherche}

De façon générale, les effets bénéfiques de la participation aux activités parascolaires sur l'engagement comportemental des élèves ont été démontrés à maintes reprises dans la littérature, et ce, autant de façon longitudinale que transversale. À l'opposé, l'absence d'activité parascolaire dans la vie académique de l'élève serait associée à un désengagement scolaire. Par contre, aucune étude n'a encore examiné le rôle modérateur du sexe et de la participation à de telles activités sur le désengagement comportemental des élèves au secondaire, selon s'ils fréquentent ou non des amis déviants. La présente étude vise donc à évaluer cet objectif auprès d'adolescents entre la première et la deuxième année du secondaire. 
II est d'abord attendu qu'en première secondaire, l'affiliation aux pairs déviants sera positivement associée au désengagement comportemental en deuxième secondaire alors qu'inversement, la participation des élèves aux activités parascolaires y sera négativement associée. De surcroît, nous anticipons que la participation aux activités parascolaires protégera les élèves qui fréquentent des amis déviants d'un éventuel désengagement. En effet, dans la mesure où les jeunes qui se retrouvent dans les activités parascolaires proviennent généralement de milieux socioéconomiques plus favorisés et sont plus susceptibles d'avoir des caractéristiques propices à la réussite scolaire (Eccles \& Barber, 1999; Fredricks \& Eccles, 2008), la fréquentation de ces groupes de jeunes dans le cadre des activités parascolaires est susceptible d'avoir une influence positive sur les adolescents à risque qui entretiennent des relations avec des pairs déviants. Enfin, parce que les filles accordent plus d'importance au contexte relationnel que les garçons (GormanSmith, 2003; Kiuru, 2007), il est attendu que le rôle protecteur de la participation aux activités parascolaires soit plus important chez les filles que chez les garçons.

\section{Méthode}

\section{Participants}

Les participants à cette étude longitudinale ont été suivis sur une période de deux ans, soit en première et en deuxième secondaire. Ces élèves proviennent de trois écoles défavorisées situées dans la grande région de Montréal. Les données utilisées dans cette étude ont été collectées sur deux temps de mesure, aux printemps de secondaire 1 ( $\mathrm{T} 1$ ) et de secondaire $2(\mathrm{~T} 2)^{1}$. Tous les élèves de secondaire 1 inscrits au régulier et quelques élèves en adaptation scolaire ont été sollicités pour participer à l'étude $(n=796)$. De ce nombre, 636 élèves ont participé au premier temps de mesure, pour un taux de participation de $79 \%$. Les élèves (49,8\% de filles) étaient alors âgés en moyenne de 12,5 ans $(\mathrm{E} . \mathrm{T} .=0,97)$. L'échantillon est ethniquement hétérogène (Caucasiens $=37,1 \%$, Asiatiques $=11,9 \%$, Noirs $=12 \%$, Latino-Américains $=5 \%$, Arabes $=12,2 \%$, Autochtones $=0,2 \%$ et autres (principalement européens) $=10,2 \%$ ). Finalement, au niveau académique, $82,5 \%$ des sujets étaient dans des classes régulières, $14 \%$ faisaient partie d'une classe d'adaptation scolaire (présence de problèmes de comportement ou d'apprentissage) et 3,4\% d'une classe de cheminement continu (soutien en mathématiques seulement).

Sur les 636 participants au T1, 448 élèves (70\% de l'échantillon initial) ont été revus l'année suivante en secondaire 2 (T2). Seuls les participants ayant des données complètes sur tous les temps de mesure ont été sélectionnés $(N=448)$. Des analyses ont été menées afin d'évaluer dans quelle mesure les sujets ayant participé aux deux temps de mesures diffèrent des sujets ayant participé au T1 sur l'ensemble des variables d'intérêts. Les résultats révèlent que les élèves n'ayant participé qu'au T1 de l'étude ne se distinguent pas des autres élèves de l'échantillon

1. En raison de la trop forte stabilité $(r=0,653)$ de la mesure de désengagement comportemental entre le T1 et le T2, nous n'avons pas contrôlé pour le désengagement comportemental au T1. Ainsi, malgré la présence de deux temps de mesure, les résultats sont de nature transversale. 


\section{2}

en regard de variables démographiques telles que l'âge, le niveau de la scolarité de la mère, l'occupation de la mère et le statut familial. Également, il n'existe pas de différence entre les groupes au niveau du désengagement comportemental $(t(290,97)=-1,079, p=0,293)$ ainsi qu'au niveau de la participation aux activités parascolaires $\left(\chi^{2}=0,997, p=0,318\right)$. Par contre, les groupes diffèrent concernant la déviance du jeune, dans la mesure où les adolescents qui font partie de l'étude sont en moyenne moins déviants que ceux qui ont quitté $(t(269,66)=-3,249, p=0,001)$. De même, les jeunes dans l'étude ont moins d'amis déviants que ceux qui sont partis $(t(282,28)=-3,58, p<0,001)$. En regard du sexe, on constate une attrition plus importante chez les garçons comparativement aux filles $\left(\chi^{2}(1)=7,369, p=0.007\right)$. Finalement, il existe une différence entre les groupes quant au type de classe; il y a plus d'élèves de classes spéciales qui ont quitté l'étude que d'élèves de classes régulières $\left(\chi^{2}=8,114, p=0,017\right)$.

\section{Procédure}

Des formulaires de consentement ont été envoyés aux parents de tous les élèves de première secondaire des trois écoles participantes. Par la suite, à chaque passation, deux assistantes de recherche formées pour le projet ont distribué le questionnaire aux élèves pour lesquels le consentement des parents avait préalablement été obtenu. Les passations ont eu lieu dans les classes où la présence du professeur était demandée afin de s'assurer du silence (souci de la confidentialité des réponses). Le niveau de scolarité de la mère, la participation aux activités parascolaires, le nombre d'amis déviants et le désengagement comportemental ont respectivement été mesurés à partir de données autorapportées à chacune des passations.

\section{Instrument de mesure}

Désengagement comportemental (T2). En deuxième secondaire, les élèves devaient rapporter la fréquence à laquelle ils manifestaient différents comportements liés au désengagement scolaire. Les réponses ont été chiffrées sur une échelle en cinq points (de 1 = presque jamais à 5 = presque toujours). Trois indicateurs d'un désengagement scolaire comportemental ont été mesurés (Archambault et al., 2009a). On retrouve deux items mesurant la manifestation de comportements perturbateurs : «À quelle fréquence déranges-tu en classe? À quelle fréquence les professeurs t'avertissent-ils parce que tu déranges en classe? "On retrouve deux items évaluant la ponctualité en classe : " À quelle fréquence arrives-tu en retard à tes cours? À quelle fréquence arrives-tu en retard à l'école? ॥. Ces deux indicateurs sont adaptés de ceux utilisés par Finn (1989; Finn \& Rock, 1997) dans leur évaluation de l'engagement scolaire. Finalement, on retrouve trois items mesurant le respect des consignes : "À quelle fréquence arrives-tu en classe sans avoir fait tes devoirs? À quelle fréquence arrives-tu sans tes livres? À quelle fréquence arrives-tu sans ton crayon ou du papier? ". Ces trois items sont adaptés des indicateurs rapportés par Rumberger et Larson (1998). Afin d'obtenir l'indice de désengagement scolaire comportemental, la moyenne des scores obtenus sur chacun des sept items a été calculée. Avec cet indice, plus la moyenne est élevée, plus les élèves rapportent un haut niveau de désengagement scolaire. L'échelle globale à un facteur a été validée à partir d'analyses factorielles 
exploratoires et confirmatoires (non publiées). Elle présente un alpha de Cronbach de 0,79 .

Nombre d'amis déviants (T1). La déviance des amis a été mesurée à partir d'une adaptation du Peer Delinquency Scale de Loeber, Farrington, Stouthamer et Kammen (1998). L'échelle des comportements antisociaux des amis comprend six items dont deux items d'agressivité physique tels que "Combien de tes amis ont poussé et bousculé physiquement d'autres jeunes? ", "Combien de tes amis ont menacé de frapper ou battre d'autres jeunes ", deux items d'agressivité relationnelle tels que "Combien de tes amis ont harcelé, écœuré verbalement d'autres jeunes ", "Combien de tes amis on fait circuler des fausses rumeurs à propos d'un ou plusieurs jeunes? " ainsi que deux items de consommation tels que "Combien de tes amis ont pris de l'alcool pour se souler? ", "Combien de tes amis ont pris de la drogue? ". Les réponses ont été évaluées sur une échelle de fréquence en cinq points (de $1=$ aucun de tes amis à $5=$ presque tous mes amis). L'échelle a été créée à partir de la moyenne des items. Elle présente un alpha de Cronbach de 0,87.

Activité parascolaire (T1). La participation aux activités parascolaires a été évaluée à partir de l'item suivant: « Depuis le début de l'année scolaire, combien d'heures par semaine consacres-tu en moyenne à des activités parascolaires (non obligatoires) qui sont organisées par l'école ou en collaboration avec l'école (sport, danse, théâtre, échecs, photo, etc.)? ॥ La réponse à cette question a été évaluée à partir d'une échelle de fréquence en six points (de 1 = je ne participe pas aux activités parascolaires à $6=$ plus de 10 heures). Avant de nous intéresser au nombre d'heures d'activités parascolaires auxquelles les jeunes participent, nous voulions vérifier si le fait d'avoir accès au contexte des activités parascolaires peut avoir un effet sur l'engagement scolaire. Puisque nous cherchions à savoir si l'élève participait ou non à une activité parascolaire, la variable a été dichotomisée. Les réponses ont été recodées 0 lorsque le participant n'a participé à aucune activité dans la dernière année (63,1 \% de l'échantillon) et 1 lorsqu'il a participé à au moins une activité parascolaire durant la dernière année à raison d'au moins 1 heure par semaine.

Sexe. La variable sexe a été évaluée à partir d'une question autorapportée recodée de la façon suivante : Garçon $=1$, Fille $=0$.

Niveau de scolarité de la mère (T1). Le niveau d'éducation maternelle étant un des meilleur indicateur proximal du niveau socioéconomique et de la réussite scolaire (Duncan \& Brooks-Gunn, 1997), les participants devaient rapporter le plus haut niveau de scolarité atteint par leurs mères : 1 = Primaire non complété, 2 = Primaire complété, 3 = Secondaire non complété, 4 = Secondaire complété, 5 = Cégep (complété ou non) 6 = Université (complétée ou non).

Déviance de l'élève (T1). La déviance du jeune a été mesurée à partir d'une adaptation du Peer Delinquency Scale de Loeber et al. (1998). L'échelle des comportements antisociaux de l'élève comprend huit items dont deux indices d'agressivité physique tels que « À quelle fréquence as-tu poussé, frappé, giflé ou donné des coups de pieds à d'autres élèves à l'école dans l'intention de faire mal 


\section{4}

à l'autre? », « Combien de fois t'es-tu battu(e) avec d'autres élèves avec l'intention de faire mal à l'autre? ", trois items d'agressivité verbale tels que "Combien de fois as-tu intimidé ou menacé de faire mal à un autre élève? ", "Combien de fois as-tu crié des noms ou dit des choses méchantes à un ou une autre élève? ", "Combien de fois as-tu ridiculisé un ou une autre élève? " ainsi que trois items de consommation de drogue ou d'alcool tels que " As-tu déjà pris de la marijuana ou du hachisch? », "As-tu déjà fait l'usage de stimulants (speed, pep pill, ecstasy, etc.) ou d'hallucinogènes (LSD, STP, PCP, mescaline...)? », « T'es-tu soulé(e) avec de la bière, du fort ou du vin? ". Les réponses ont été chiffrées sur une échelle de fréquence en six points ( $1=$ jamais, $2=1$ ou 2 fois, 3 = parfois, de temps en temps, 4 = environ 1 fois par semaine, 5 = quelques fois par semaine, $6=$ tous les jours). L'échelle a été créée à partir de la moyenne des items. Elle présente un alpha de Cronbach de 0,87 .

\section{Stratégie analytique}

Nous avons d'abord examiné l'intercorrélation entre chacune des variables à l'étude. Afin d'évaluer dans quelle mesure la déviance des amis est associée au désengagement comportemental ultérieur, une analyse de régression multiple hiérarchique a ensuite été effectuée. Les prédicteurs ont été analysés en quatre étapes consécutives. Dans un premier temps, la scolarité de la mère et la déviance du jeune au T1 ont été considérées simultanément comme variables de contrôle (modèle 1). Dans un deuxième temps, le nombre d'amis déviants (modèle 2), la participation aux activités parascolaires et le sexe (modèle 3 ) ont été ajoutés au modèle. Enfin, les effets d'interaction doubles et triple impliquant le sexe, le nombre d'amis déviants et la participation aux activités parascolaires ont été considérés séparément, puis de façon simultanée dans l'analyse. Par contre, seule la dernière étape qui où toutes les interactions ont été testés simultanément sera présentée (modèle 4). Ces interactions ont été créées et analysées à partir de la méthode proposée par Aiken et West (1991). Selon cette méthode, toutes les variables doivent préalablement être centrées autour de la moyenne afin d'éviter les problèmes de multicolinéarité. On crée ensuite les variables d'interaction en multipliant la variable indépendante et les variables modératrices. Advenant un effet d'interaction significatif, la relation entre un prédicteur et le désengagement scolaire des élèves au T2 est alors estimée à différents niveaux du modérateur.

\section{Résultats}

\section{Analyses exploratoires}

Dans un premier temps, des analyses corrélationnelles ont été menées afin d'examiner les relations entre les différentes variables à l'étude, soit la scolarité de la mère, la déviance du jeune, la participation aux activités parascolaires, le nombre d'amis déviants et le désengagement comportemental au T2. Le tableau 1 présente les moyennes, les écarts-types et les intercorrélations qui existent entre ces variables. On constate à l'examen de ce tableau que toutes les variables corrèlent de façon positive et significative avec le désengagement comportemental de l'élève, à l'exception de la scolarité de la mère qui est négativement associée au désengagement $(p<0,05)$. La direction des relations entre les variables suggère 
que les élèves les plus désengagés sont ceux de sexe masculin, les élèves qui ont une mère peu scolarisée, qui présentent des comportements déviants, qui ont des amis déviants et qui participent à des activités parascolaires. Selon les critères de Cohen (1988), la plupart des corrélations entre les variables sont faibles ou modérées. Par ailleurs, on note que le nombre d'amis déviants rapportés par l'élève est très fortement associé à la déviance du jeune au T1 $(r=0,66)$ et modérément associé au désengagement comportemental $(r=0,28)$. On constate également l'absence de multicolinéarité puisqu'aucune variable n'est corrélée au-delà de 0,70. Finalement, on voit que le sexe et la participation aux activités parascolaires ne sont corrélés avec aucune autre variable que le désengagement comportemental de l'élève.

Tableau 1. Intercorrélations entre les variables à l'étude

\begin{tabular}{lcccccc}
\hline Variables & 1 & 2 & 3 & 4 & 5 & 6 \\
\hline $\begin{array}{l}\text { 1. Scolarité de la mère } \\
\text { 2. Déviance de l'élève }\end{array}$ & 0,01 & & & & & \\
$\begin{array}{l}\text { 3. Participation aux activi- } \\
\quad \text { tés parascolaires }\end{array}$ & 0,04 & 0,03 & & & & \\
$\begin{array}{l}\text { 4. Sexe (Garçons) } \\
\text { 5. Nombre d'amis déviants }\end{array}$ & 0,03 & 0,09 & 0,07 & & & \\
$\begin{array}{l}\text { 6. Désengagement } \\
\quad \text { comportemental }\end{array}$ & $-0,10^{*}$ & $0,23^{* *}$ & $0,09^{*}$ & $0,10^{*}$ & $0,28^{* *}$ & \\
& & & & & & \\
$M$ & 5,24 & 1,25 & 0,36 & 0,47 & 1,34 & 1,81 \\
ÉT & 1,26 & 0,50 & 0,48 & 0,50 & 0,49 & 0,68 \\
\hline
\end{tabular}

Notes. ${ }^{*} p<.05 ;{ }^{* *} p<.01 ;{ }^{* * *} p<.001$

$\mathrm{M}=$ moyenne, $\mathrm{É}^{\mathrm{T}}=$ écart-type

\section{Modèles de prédiction du désengagement comportemental}

Afin d'évaluer les hypothèses de l'étude, une analyse de régression hiérarchique a ensuite été effectuée. Le tableau 2 présente les résultats obtenus à la suite de chacune des quatre étapes. Le modèle 1 indique d'abord que la scolarité de la mère et la déviance de l'élève au T1 permettent d'expliquer $6,4 \%$ de la variance du désengagement comportemental $(F(2,445)=15,30, p<0,001)$. Les deux covariables contribuent de façon unique et indépendante à rendre compte du désengagement comportemental. On constate que moins la mère est scolarisée et plus l'élève est déviant, plus il est désengagé en secondaire 2. En ajoutant ensuite le nombre d'amis déviants, le modèle 2 permet d'expliquer $2,6 \%$ de variance supplémentaire $(F(1,444)=12,64, p<0,001)$. Cette variable contribue donc de façon unique et indépendante à rendre compte du désengagement comportemental de l'élève au T2. Ainsi, plus l'élève a d'amis déviants en secondaire 1, plus il 


\section{6}

est désengagé en secondaire 2. On remarque toutefois que dans ce modèle, la déviance de l'élève cesse de contribuer de manière unique et indépendante à rendre compte du désengagement scolaire au T2. Le troisième modèle indique que l'ajout de la participation aux activités parascolaires et du sexe permet d'accroître de façon significative le pourcentage de variance expliquée $(F(2,442)=3,38$, $p=0,035)$ de $1,4 \%$. Par contre, la contribution directe de chacun des modérateurs à l'explication du désengagement comportemental demeure modeste et seulement marginalement significative. Plus spécifiquement, les résultats indiquent que les garçons et les élèves qui participent aux activités parascolaires sont marginalement plus désengagés au temps 2 .

Tableau 2. Modèles de régression linéaire multiple hiérarchique visant à prédire le désengagement.

\begin{tabular}{lcccc}
\hline & Modèle 1 & Modèle 2 & Modèle 3 & Modèle 4 \\
\cline { 2 - 5 } & $\beta$ & $\beta$ & $\beta$ & $\beta$ \\
\hline Scolarité de la mère & $-0,10^{*}$ & $-0,08$ & $-0,08+$ & $-0,07$ \\
Déviance de l'élève & $0,24^{* * *}$ & 0,09 & 0,08 & 10,1 \\
Nombre d'amis déviants & & $0,22^{* * *}$ & $0,21^{* * *}$ & $0,41^{* * *}$ \\
$\begin{array}{l}\text { Participation aux activités } \\
\text { parascolaires }\end{array}$ & & $0,08+$ & 0,06 \\
Sexe & & & & $0,06+$ \\
Sexe X Activités \\
parascolaires
\end{tabular}

Note. ${ }^{*} p<0,05 ;{ }^{* *} p<0,01 ;{ }^{* *} p<0,001 ;+p<0,10$ 
Finalement, l'ajout des effets d'interaction doubles et triples impliquant le sexe, le nombre d'amis déviants et la participation aux activités parascolaires au modèle 4 permet d'expliquer $3,1 \%$ de variance supplémentaire $(F(4,438)=3,966$, $p=0,004)$. Ce modèle indique d'abord que l'interaction entre le sexe des participants et la participation aux activités parascolaires s'avère non significative. Par contre, l'interaction entre le nombre d'amis déviants et la participation aux activités parascolaires s'avère significative, bien que de façon marginale. Nous avons tout de même décidé de la décomposer selon la méthode proposée par Aiken et West (1991). À la suite de cette décomposition, on constate que le nombre d'amis déviants est positivement associé au désengagement scolaire ultérieur chez les élèves qui ne participent pas aux activités parascolaires $(\beta=0,257, p=0,04)$. Par contre, chez ceux qui participent à une activité, le nombre d'amis déviant n'est pas associé au désengagement $(\beta=-0,081, p=0,536)$. Par ailleurs, nos résultats indiquent également qu'il existe un effet d'interaction significatif entre le sexe des participants et le nombre d'amis déviants. De manière plus spécifique, cet effet suggère que le nombre d'amis déviants est positivement associé au désengagement scolaire ultérieur chez les filles $(\beta=1,195, p<0,001)$, mais pas chez les garçons $(\beta=0,084$, $p=0,268$ ).

Finalement, l'effet triple incluant le nombre d'amis déviants, le sexe et la participation aux activités parascolaires s'avère également significatif pour expliquer le désengagement comportemental au temps 2 . Plus spécifiquement, les analyses subséquentes indiquent que chez les garçons, la déviance des amis n'est pas associée au désengagement et ce, qu'ils participent $(\beta=0,226, p=0,181)$ ou qu'ils ne participent pas aux activités parascolaires $(\beta=-0,053, p=0,675)$. Par contre, le portrait observé chez les filles est assez différent. En effet, la fréquentation d'amis déviants est associée à une augmentation significative du désengagement $(\beta=0,571, p<0,001)$ chez les filles qui ne participent aux activités parascolaires. Cependant, la déviance des amis n'est pas associée au désengagement chez celles qui participent aux activités scolaires $(\beta=0,223, p=0,175)$. Conséquemment, la participation à une activité parascolaire agit comme facteur de protection chez les filles affiliées à des amis déviants dans la mesure où il annule l'impact des amis des déviants sur le désengagement.

\section{Discussion}

À l'adolescence, les relations avec les pairs jouent un rôle important dans la formation de l'identité. Les jeunes s'observent et s'influencent mutuellement, ce qui affecte tant positivement que négativement leurs comportements, leurs attitudes et leurs habiletés (Rubin, Bukowski, Parker, \& Bowker, 2008). Dans ce sens, plusieurs études ont démontré l'influence négative que peut avoir la fréquentation de pairs déviants sur l'engagement scolaire des jeunes (Stanard et al., 2010). À l'inverse, d'autres auteurs suggèrent que la participation à une activité parascolaire pourrait offrir une opportunité aux adolescents à risque de vivre une expérience positive à l'école, ce qui favoriserait leur engagement et leur réussite académique (Brown \&Theobald, 1998; Eccles et al., 2005; Gilman et al., 2004; Marsh, 1992). Ainsi, la présente étude avait comme objectif d'évaluer dans quelle mesure la participation aux activités parascolaires peut agir comme facteur de protection dans la relation 


\section{8}

entre l'affiliation à des pairs déviants et le désengagement comportemental chez les adolescents et adolescentes, entre la première et la deuxième secondaire.

En lien avec les études antérieures (Dishion, Andrews, \& Crosby, 1995; Stanard et al., 2010), notre première hypothèse soutenait que l'affiliation à des pairs déviants en première secondaire serait positivement associée au désengagement comportemental en deuxième secondaire. Nos résultats appuient cette idée. En effet, les adolescents qui ont plusieurs amis déviants en première année du secondaire sont également ceux qui rapportent le plus haut niveau de désengagement l'année suivante. De plus, la déviance des amis et de l'élève étant très fortement associées, ces élèves semblent aussi présenter davantage de comportements déviants. Puisque les comportements de délinquance et de rébellion scolaire sont souvent le reflet d'un même syndrome général de déviance (Jessor \& Jessor, 1977), à travers différents mécanismes de socialisation et de cohésion, le fait d'avoir des amis qui présentent des comportements déviants pourrait donc non seulement contribuer à l'accroissement des comportements antisociaux du jeune, mais également à son désengagement ultérieur.

Nous anticipions également que la participation des élèves aux activités parascolaires en première secondaire serait négativement associée à leur désengagement comportemental en deuxième secondaire. Les résultats obtenus infirment cette hypothèse. En effet, la relation simple qui existe entre la participation aux activités parascolaires et le désengagement est positive, signifiant que plus l'élève participe aux activités parascolaires, plus il est désengagé. De plus, après avoir contrôlé pour la déviance de l'élève et de ses amis, cet effet négatif des activités parascolaires persiste, mais devient marginal ce qui suggère que, déviants ou non, les élèves qui s'investissent au sein de ces activités ont davantage tendance à déranger en classe, arriver en retard à leurs cours et à ne pas avoir le matériel nécessaire pour faire leur travail correctement. Vu les nombreuses études qui démontrent les effets bénéfiques de la participation aux activités parascolaires sur les comportements, l'engagement et la participation des jeunes à l'école (Beiswenger \& Grolnick, 2010; Eccles \& Fredricks, 2006; Eccles \& Templeton, 2002), un tel résultat s'avère assez surprenant. II existe toutefois certaines pistes d'explication.

Tout d'abord, il est possible qu'un certain nombre d'activités auxquelles les jeunes participent soient plus positivement associées au désengagement que d'autres. En effet, selon plusieurs auteurs, des activités telles que les sports d'équipe offriraient aux jeunes un contexte favorable à l'émergence et au développement de comportements déviants (Borden, Donnermeyer, \& Scheer, 2001; Eccles et al., 2003; Gardner et al., 2009; Lansford, 2006). À l'intérieur de certaines de ces activités, il existerait en effet une sous-culture valorisant à la fois la réussite scolaire, mais aussi l'adoption de comportements déviants tels que la consommation d'alcool, une tendance à faire la fête, etc., qui pourrait avoir un impact sur le désengagement scolaire de l'élève. Comme la nature des activités parascolaires auxquelles les jeunes participaient était non spécifiée dans cette étude, nous n'avons pu vérifier l'influence différentielle de ce facteur sur le désengagement. II s'agit toutefois d'une piste d'explication qui serait intéressante à explorer dans de futurs travaux. 
La participation à des activités parascolaires moins structurées, soit celles peu supervisées par les adultes, pourrait également contribuer au désengagement des élèves en favorisant l'émergence de comportements de non-conformisme ou de déviance, souvent mineurs, qui sont caractéristiques de l'adolescence (Agnew \& Petersen, 1989; Osgood et al., 1996; Mahoney, 2000). Dans la présente étude, aucune donnée ne permet de documenter le niveau d'encadrement des activités parascolaires, laissant ainsi présumer qu'il pourrait s'agir d'activités moins structurées et où la présence d'adolescents plus déviants aurait peu avoir un impact négatif sur le désengagement scolaire de l'élève. Par contre, cela demeure une piste à explorer.

Par ailleurs, l'association positive entre la participation aux activités parascolaires et le désengagement pourrait aussi être expliquée par la nature de notre échantillon. Les participants de notre étude faisaient partie d'écoles en milieu défavorisé seulement. Or, la plupart des études qui mentionnent les effets bénéfiques de la participation aux activités parascolaires disposent de sujets de différents statuts socio-économiques, ou encore de participants provenant de milieux favorisés. Or, il est possible que dû au biais de sélection, l'effet attendu des activités parascolaires soit inexistant parce que les activités sont moins disponibles ou populaires en milieux défavorisés. De plus, dans certains de ces milieux, on incite les élèves à risque à participer à des activités parascolaires pour soutenir leur persévérance et leur réussite à l'école. Ainsi, puisque le désengagement des élèves au T1 n'a pas été contrôlé, il est plausible que les élèves plus désengagés à la base aient été plus nombreux à participer à des activités dans notre étude. Enfin, rappelons une fois de plus qu'après avoir contrôlé pour la déviance de l'élève et de ses pairs, les activités parascolaires prédisent seulement de façon marginale le désengagement scolaire. Ce résultat suppose donc que l'affiliation à des pairs antisociaux et la déviance du jeune permettent davantage de prédire le désengagement comportemental que la participation aux activités parascolaires en soi. Nos résultats doivent donc être interprétés dans ce sens.

Ceci étant dit, il était également attendu que la participation aux activités parascolaires agisse à titre de facteur de protection sur le lien entre l'affiliation à des pairs déviants et le désengagement scolaire des élèves. Nos résultats soutiennent partiellement cette idée. En effet, l'influence protectrice de la participation aux activités parascolaires existe, mais uniquement chez les filles. Ainsi, bien que les adolescentes qui ont plus d'amis déviants soient plus désengagées au niveau comportemental, c'est-à-dire qu'elles respectent moins les règles et s'impliquent moins dans les activités proposées en classe, cela est uniquement vrai chez celles qui ne participent pas aux activités parascolaires. Chez celles qui y participent, il n'existe aucun lien entre la déviance des amis et le désengagement. Dans ce sens, nos résultats démontrent que la participation aux activités parascolaires agit à la fois comme facteur pouvant marginalement influencer le désengagement, mais également comme facteur de protection chez les filles du fait que chez celles qui participent à ce type d'activités, les risques associés à la fréquentation d'amis déviants disparaissent. Encore une fois, ceci pourrait laisser sous-entendre que les filles qui ne participent pas aux activités parascolaires et qui ont des amis déviants présentent certaines caractéristiques communes défavorables à l'engagement. Par contre, cela pourrait également signifier que chez les filles qui ont des amis déviants, 
la participation à une activité parascolaire offre un contexte positif dans lequel les adolescentes peuvent être exposées à des pairs prosociaux (Agnew \& Peterson, 1989; Mahoney, 2000; Osgood et al., 1996), ce qui favoriserait leur engagement. Parce que les filles accordent généralement plus d'importance aux relations d'amitié que les garçons (Gorman-Smith, 2003; Kiuru, 2007), l'exposition des filles qui ont des amis déviants à des activités parascolaires au sein desquelles elles peuvent créer de nouveaux liens avec des pairs prosociaux semble contrecarrer l'influence négative qu'exercent les amis antisociaux.

Le portrait des garçons est toutefois assez différent. En effet, bien que les garçons soient généralement plus désengagés que les filles, nos résultats indiquent que leur désengagement scolaire ne semble pas être influencé par la déviance de leurs amis ou par leur investissement au sein d'activités parascolaires. II faut donc chercher une autre piste d'explication. Dans ce sens, nous postulons que les garçons qui manquent l'école, arrivent en retard et dérangent en classe puissent eux-mêmes présenter des comportements déviants et que ces comportements ont une influence importante, qui outrepasse celle de leurs amis ou des activités parascolaires, sur leur désengagement. Plusieurs études démontrent en effet que comparativement aux filles, les garçons sont plus enclins à adopter des comportements à risque (c.à-d. impulsivité, agressivité, hyperactivité), à être déviants, à moins aimer l'école et y être plus désengagés (Barber, 1996; Cox, 2000; Hatzichristou \& Hopf, 1996). Ainsi, le désengagement des garçons pourrait être davantage influencé par leurs propres comportements antisociaux que par les comportements de leurs amis et le fait de participer ou non à une activité parascolaire n'influencerait pas cette relation.

\section{Implications pour la recherche}

La présente étude a démontré que la participation à une activité parascolaire peut jouer à la fois le rôle de facteur risque, mais également de facteur de protection sur le désengagement scolaire des filles qui ont des amis déviants. À la lumière de ces résultats, l'idée n'est donc pas de proscrire la participation à ce type d'activités, mais plutôt de continuer les recherches afin de mieux comprendre la nature des activités parascolaires pouvant favoriser ou nuire à l'engagement des garçons et des filles. Pour ce faire, des études sont nécessaires afin de comparer l'effet différentiel de différents types activités tels que les sports d'équipes, les sports individuels, l'art, le bénévolat, les activités intellectuelles ou de culture générale sur l'engagement des adolescents. Également, il serait important que d'autres recherches se concentrent sur les caractéristiques individuelles des élèves qui participent aux activités, notamment à l'effet de la popularité, ainsi que sur les activités auxquelles participent les jeunes à risque, afin d'identifier celles au sein desquelles ils sont le plus susceptibles de retrouver des pairs qui agiront comme modèle positif. Enfin, il serait également important de s'intéresser de manière plus spécifique aux activités auxquelles participent les garçons et les filles. En effet, il est plausible que les adolescents et adolescentes s'investissent dans des activités différentes, avec une intensité différente, et qu'en l'occurrence, ces distinctions influencent de manière spécifique leur engagement scolaire. Finalement, dans la mesure où la plupart des adolescents se côtoient sur une période de cinq ans en contexte scolaire, il serait pertinent de vérifier l'effet cumulatif de la participation aux activités parascolaires dans le temps, afin de voir s'il existe des variations en 
fonction de l'âge ou de la force du lien d'attachement qui unit les participants à une même activité sur une longue période.

\section{Implications pour l’intervention psychoéducative}

Bien que les mécanismes qui sous-tendent l'influence négative de la participation aux activités parascolaires sur l'engagement méritent d'être explorés davantage, nos résultats suggèrent qu'avant même de planifier, d'organiser et d'instaurer une banque d'activités parascolaires, une première avenue pour l'intervention psychoéducative serait l'observation et l'évaluation des besoins des jeunes qui participeront à ces activités. Cette démarche permettra ainsi de comprendre qui sont les élèves susceptibles d'y participer, leurs motivations, leurs compétences, leurs difficultés et le niveau d'encadrement et de supervision à envisager. Cette évaluation préalable qui devrait se maintenir en cours d'activité favoriserait la mise en place et le maintien de conditions favorables à la planification, l'organisation et l'animation d'activités parascolaires adaptées à la clientèle. Par exemple, pour une école en milieu défavorisé, l'implantation d'activités très structurées, notamment par la supervision d'adultes compétents, l'application d'un code de règles et procédures rigoureux de même que d'un système de responsabilité et de récompenses, permettrait à la fois l'amélioration de la qualité de vie de l'école, mais également l'atteinte des objectifs de prévention souhaités. Dodge et al. (2006) proposent également des pistes de solutions pour éviter le processus d'entraînement à la déviance présent dans certains groupes sociaux en milieux scolaires. Une des solutions intéressantes dans un contexte parascolaire consiste à intégrer des jeunes bien adaptés et légèrement plus âgés à titre de coanimateurs. Par exemple, en impliquant des élèves prosociaux et engagés de secondaire trois ou quatre comme coanimateurs d'une troupe de théâtre ou de danse de secondaire un, ces jeunes pourront agir comme modèles favorables à l'engagement.

Finalement, tel que mentionné ci-haut, nos résultats ont démontré que l'influence de la participation aux activités parascolaires n'est pas la même pour les garçons et les filles. Ainsi, en considérant que les filles sont plus vulnérables à l'influence de leurs pairs et particulièrement celles qui ont des amis déviants, il serait souhaitable que l'on puisse éviter la présence de sous-culture déviante dans certaines activités parascolaires de même que la présence de leaders négatifs qui sont susceptibles d'influencer les comportements de ces adolescentes et ainsi augmenter leur désengagement. Pour ce faire, il serait intéressant de considérer la possibilité de faire une présélection d'individus selon des critères spécifiques avant le début des activités, ou encore d'établir des conditions d'admission différentes pour chacune des participantes selon des objectifs précis. Quant aux garçons, dans l'option où leurs propres comportements déviants puissent constituer un risque, il serait souhaitable de cibler les adolescents qui présentent des trajectoires déviantes persistantes dès leur arrivée au secondaire afin de les intégrer à des programmes de prévention des problèmes de comportement et de délinquance. De plus, il serait probablement bénéfique pour ces jeunes que la participation à des activités parascolaires soit plus encadrée et structurée à l'aide d'objectifs précis, plutôt qu'offerte comme une simple activité parascolaire ludique. 
Forces et Limites

Cette étude comporte plusieurs forces. D'abord, elle constitue l'une des rares études ayant permis de vérifier l'influence de la participation aux activités parascolaires sur le désengagement comportemental des adolescents et des adolescentes. De plus, le fait d'utiliser des items de délinquance masculine et féminine (c.-à-d., agressivité directe et indirecte, agressivité verbale) afin de mesurer la déviance de l'élève et des pairs a permis une meilleure compréhension des différences sexuelles dans la relation entre l'affiliation à des pairs déviants et le désengagement comportemental des adolescents. Cette recherche contribue donc de manière significative à la littérature émergente sur ce sujet.

Malgré son caractère novateur, cette étude présente cependant certaines limites. Tout d'abord, la généralisation des résultats devrait être limitée aux écoles urbaines francophones de milieux défavorisées. Deuxièmement, dans la mesure où la nature de l'activité, le nombre d'activités, leur intensité, les caractéristiques des jeunes qui y participent et des adultes qui les supervisent sont susceptibles d'influencer les relations à l'étude, le manque d'information à cet effet est certainement une limite de l'étude. Troisièmement, tel qu'indiqué dans la section méthode, plusieurs jeunes, probablement plus à risque d'être désengagés, ont quitté l'étude. Comme il n'y a pas eu d'imputation faite sur les données manquantes, cette attrition a pu influencer nos résultats. Par exemple, il est possible que la participation à une activité parascolaire ait un impact plus positif sur l'engagement scolaire d'élèves à risque. De plus, toutes les données ont été recueillies par une seule source, à savoir l'adolescent lui-même, ce qui a certainement pu influencer les résultats. En effet, le fait d'avoir qu'une seule source peut limiter la validité de la mesure du fait que la perception possiblement biaisée de certains adolescents sur leurs comportements déviants ou ceux de leurs amis a certainement pu avoir une influence sur les données recueillies. Dans ce sens, il aurait été bénéfique d'obtenir l'évaluation d'un l'adulte (professeur, parent, etc.), ou encore d'utiliser une mesure sociométrique afin d'améliorer la validité de la mesure. Enfin, en raison d'une trop forte stabilité de la mesure, le désengagement comportemental au T1 n'a pas été contrôlé. Ainsi, malgré la présence de deux temps de mesure, les résultats de l'étude doivent être interprétés de manière transversale.

\section{Conclusion}

En conclusion, la présente étude a démontré que les activités parascolaires peuvent agir à la fois à titre de facteur de risque, mais également de facteur de protection contre le désengagement des filles qui ont des amis déviants. Par conséquent, il est primordial de pouvoir offrir à ces adolescentes des conditions en milieux scolaires qui pourront assurément les protéger contre la délinquance de leurs amis. Dans la mesure où la psychoéducation se spécialise à la fois dans l'intervention auprès des jeunes en difficultés d'adaptation de même que dans l'animation d'activités de groupe, le psychoéducateur a certainement un rôle à jouer dans l'amélioration des conditions gagnantes pour que les activités parascolaires puissent contribuer à l'engagement et la réussite scolaire des jeunes. 


\section{Références}

Ablard, K., \& Lipschultz, R. E. (1998). Selfregulated learning in high-achieving students: Relations to advanced reasoning, achievement goals and gender. Journal of Educational Psychology, 90 (1), 94-101.

Agnew, R., \& Peterson, D. M. (1989). Leisure and delinquency. Social Problems, 36(4), 332-350.

Aiken, L. S., \& West, S. G. (1991). Multiple regression: Testing and interpreting interactions. Newbury Park, CA: Sage.

Appleton, J., Christenson, S., Dongjin, K., \& Reschly, A. (2006). Measuring cognitive and psychological engagement: Validation of the Student Engagement Instrument. Journal of School Psychology, 44(5), 427-445. doi: http://dx.doi.org/ 10.1016/j.jsp.2006.04.002.

Archambault, I., Janosz, M., Fallu, J. S., \& Pagani, L. S. (2009a). Student engagement and its relationship with early high school dropout. Journal of Adolescence, 32(3), 651-670. doi: http://dx.doi.org/ 10.1016/j.adolescence.2008.06.007

Archambault, I., Janosz, M., Morizot, J., \& Pagani, L. S. (2009b). Adolescent behavioral, affective, and cognitive engagement in school: Relationship to dropout. Journal of School Health, 79, 402-409.

Bandura, A. (1986). Social foundations of thought and action: A social cognitive theory. Englewood Cliffs, N.J.: PrenticeHall.

Barber, M. (1996). The learning game: Arguments for an education revolution. London: Victor Gollancz.

Barber, B. L., \& Eccles, J. S. (1997). Student council, volunteering, basketball, or marching band: What kind of extracurricular involvement matters? Paper presented at the biennial meeting of the Society for Research on Child Development, Washington, DC. doi: 10.1177/0743558499141003.
Barber, B. L., Eccles, J. S., \& Stone, M. R. (2001). Whatever happened to the jock, the brain and the princess? Young adult pathways linked to adolescent activity involvement and social identity. Journal of Adolescent Research, 16, 429-455. doi: $10.1177 / 0743558401165002$.

Barber, B. L., Stone, M. R., Hunt, J., \& Eccles, J. S. (2005). Benefits of activity participation: The roles of identity affirmation and peer group norm sharing. Dans J. L. Mahoney, R. W. Larson, \& J. $\mathrm{S}$. Eccles (dir). Organized activities as contexts of development: Extracurricular activities, after-school and community programs (pp. 185-210). Mahwah, NJ: Erlbaum.

Beiswenger, K. L., \& Grolnick, W. (2010). Interpersonal and intrapersonal factors associated with autonomous motivation. Journal of Early Adolescence, 30(3) 369394. doi: 10.1177/0143034391123010.

Becker, H. (1985). Outsiders: Études de sociologie de la déviance. Paris, Métailié.

Benenson, J.F., \& Schinazi, J. (2004). Sexe differences in reactions to outperforming same-sex friend. British Journal of Developmental Psychology, 22, 317334.

Borden, L. M., Donnerymeyer, J. F., \& Scheer, S. D. (2001). The influence of extra-curricular activities and peer influence on substance use. Adolescent and Family Health, 2, 12-19. doi: $10.3102 / 00346543075002159$.

Bronfenbrenner, U. (1979). The Ecology of Human Development: Experiments by nature and design. Cambridge, MA: Harvard University Press.

Brown, B. B., \& Theobald, W. (1998). Learning contexts beyond the classrooms: Extracurricular activities, community organizations, and peers groups. Dans K. Borman et B. Schneiders (dir), The adolescent years: Social influences and educational challenges (p.109-141). Chicago, IL: The university of Chicago Press. 
Burk, W. J., Kerr, M., \& Stattin, H. (2008). The co-evolution of early adolescent friendship networks, school involvement, and delinquent behaviors. Revue Française de Sociologie, 49(3), 499522.

Burk W. J., Steglich C. E. G., \& Snijders T. A. B. (2007). Beyond dyadic interdependence: Actor-oriented models for co-evolving social networks and individual behaviors. International Journal of Behavioral Development, 31, 4, 397-404. doi: $10.1177 / 0165025407077762$.

Cillessen, A. H. (2011). Toward a theory of popularity. Dans A. H. Cillessen, D. Schwartz et L. Mayeux (dir.), Popularity in the peer system (pp.273-299). New York, NY: Guilford Press.

Cillessen, A.H.N., \& Mayeux, L. (2007). Expectations and perceptions at school transitions: The role of peer status and aggression. Journal of School Psychology, 45, 567-586.

Cohen, A. R. (1958). Upward communication in experimentally created hierarchies. Human Relations, 11, 41-54.

Cohen, J. (1988). Statistical power analysis for the behavioral sciences (2nd ed.). New Jersey: Lawrence Erlbaum.

Coleman, J. S. (1961). Social Climates in High Schools. U.S Dept. Of Health, Education, and Welfare, Office of Education.

Connell, J. P. (1990). Context, self, and action: A motivational analysis of selfsystem processes across the lifespan. Dans D. Cicchetti (dir), The self in transition: Infancy to childhood (pp. 61-97). Chicago: University of Chicago Press.

Cook, T. D., Herman, M. R., Phillips, M., \& Settersen, R. A., Jr. (2002). Some ways in which neighborhoods, nuclear families, friendship groups, and schools jointly affect changes in early adolescent development. Child Development, 73, 1283-1309.

Covay, E., \& Carbonaro., W. (2010). Beyond school walls: Participation in extracurricular activities, classroom behavior, and academic achievement. Sociology of Education, 83, 20-45.
Cox, T. (2000). Pupils' perspectives on their education. Dans T. Cox (dir.), Combating educational disadvantage. Meeting the needs of vulnerable children. London: Falmer Press.

Cusson, M. (1992). Déviance. Dans R. Boudin (dir.), Traité de sociologie. France : Paris.

Damon, W., \& Hart, D. (1988). Selfunderstanding in childhood and adolescence. NewYork, NJ: Cambridge University Press.

Denault, A.S., \& Poulin, F. (2011). Peer deviancy in the activity peer group and youths' problem behaviors: A look at moderating effects. Canadian Journal of Behavioural Science, 44, 83-92.

Darling, N. (2005). Participation in extracurricular activities and adolescent adjustment: Cross-sectional and longitudinal findings. Journal of Youth and Adolescence, 34, 493-505. doi: 10.1007/ s10964-005-7266-8

Dishion, T. J., Andrews, D. W., \& Crosby, L. (1995). Antisocial boys and their friends in early adolescence: Relationship characteristics, quality, and interactional process. Child Development, 66 (1), 139-151.

Dishion, T. J., \& Patterson, G. R. (1997). The timing and severity of antisocial behavior: Three hypotheses within an ecological framework. Dans D., Stoff, J., Brieling, \& J. Maser (dir.), Handbook of antisocial behavior (p.205-217). New York, NY: John Wiley \& Sons.

Dodge, K., Lansford, J. E., \& Dishion, T. J. (2006). Findings and recommendations: A blueprint to minimize deviant peer influence in youth interventions and programs. Dans K. A. Dodge, T. J. Dishion, \& J. E. Lansford (dir.), Deviant peer influences in programs for youth (pp. 366-394). New York, NY: Guilford.

Dotterer, A. M., McHale, S. M., \& Crouter, A. C. (2007). Implications of out-of-school activities for school engagement in African American adolescents. Journal of Youth Adolescence, 36, 391-401. 
Dubow, F., Rowell Huesmann, L., Boxer, P., Pulkkinen, L., \& Kokko, K. (2006). Middle childhood and adolescent contextual and personal predictors of adults educational and occupational outcomes: A mediational model in two countries. Developmental Psychology, 42, 937-949.

Duncan, G.J. et Brooks-Gunn, J. (1997). Consequences of growing up poor. New York, NY: Russell Sage. Foundation.

Eccles, J. S., \& Barber, B. L., (1999). Student council, volunteering, basketball, or marching band: What kind of extracurricular involvement matters? Journal of Adolescence Research, 14,10-43

Eccles, J. S., Barber, B. L., Stone, M., \& Hunt, J. (2003). Extracurricular activities and adolescent development. Journal of Society Issues, 59, 865-889. doi:10.104 6/j.0022-4537.2003.00095.

Eccles, S. J., \& Fredricks, J. A. (2006). Is extracurricular participation associated with beneficial outcomes? Concurrent and longitudinal relations. Developmental Psychology, 42(4), 698713. doi: 10.1037/0012-1649.42.4.698

Eccles, J. S., \& Templeton, J. (2002). Extracurricular and other afterschool activities for youth. Review of Education, 26, 113-180. doi: $10.3102 / 0091732 \times 026001113$

Fallu, J.-S., Brière, F. N., Vitaro, F., Cantin, S., \& Borge, A. I. H. (2011). The Influence of close friends on adolescent substance use: Does popularity matter? Dans A. Ittel, H. Merkens, \& L. Stecher (dir). Jahrbuch Jugendforschung (pp. 235262).

Fauth, R. C., Roth, J. L., \& Brooks-Gunn, J. (2007). Does the neighborhood context alter the link between youth's afterschool time activities and developmental outcomes? A multilevel analysis. Developmental Psychology, 43, 760777.

Feldman, A. M., \& Matjasko, J. L. (2005). The role of school-based extracurricular activities in adolescent development: A comprehensive review and future direc- tions. Review of Educational Research, 75,159-210.

Fredricks J. A., \& Eccles, J. (2008) Participation in extracurricular activities in the middle school years: Are there developmental benefits for African American and European American youth? Journal of Youth Adolescence, 37, 1029-1043.

Fredricks, J. A., Blumenfeld, P. C., \& Paris, A. H. (2004). School engagement: Potential of the concept, state of the evidence. Revue of Educational Research, 74, 59109. doi: 10.3102/00346543074001059

Finn, J. D. (1989). Withdrawing from school. Review of Educational Research, 59, 117-142.

Finn, J. D., \& Cox, D. (1992). Participation and withdrawal among fourth grade pupils. American Educational Research Journal, 29(1), 141-162.

Finn, J. D., \& Rock, D. (1997). Academic success among students at risk for school failure, Journal of Applied Psychology, 82, 221-234. doi: 10.1037/0021-9010. 82.2.221

Furrer, C., Skinner, E., Marchand, G., \& Kindermann, T. (2006). Engagement versus disaffection as central constructs in the dynamics of motivational development. Biennial Meeting for the Society for Research on Adolescence, San Francisco, CA. doi: 10.1037/a0012840

Gardner, M., Roth, J. L., \& Brooks-Gunn, J. (2009). Sports participation and juvenile delinquency: The role of the peer context among adolescent boys and girls with varied histories of problem behavior. Developmental Psychology, 45, 341353. doi: $10.1037 / \mathrm{a} 0014063$

Gilman, R., Meyers, J., \& Perez, L. (2004). Structured extracurricular activities among adolescents: A review of the literature for school psychologists. Psychology in the Schools, 1, 31-41. doi: 10.1002/pits.10136 
Gorman-Smith, D. (2003). Prevention of antisocial behavior in females. Dans D.P. Farrington and J. Coid (dir.), Primary prevention of antisocial behavior (pp. 292-317). Cambridge, UK: Cambridge University Press.

Haselager, G. J., Hartup, W. W., van Lieshout, C. F., \& Riksen-Walraven, J. M. (1998). Similarities between friends and nonfriends in middle childhood. Child Development, 69, 1198-1208.

Hatzichristou, C., \& Hopf, D. (1996). A multiperspective comparison of peer sociometric status groups in childhood and adolescence. Child Development, 67, 1085-1102.

Hartup, W. W. (1996). The company they keep: Friendships and their developmental significance. Child Development, 67, 1-13.

Haynie, L., \& Osgood,D, W. (2005). Reconsidering peers and delinquency: How do peers matter? Social Forces, 84(2), 1109-1130.

Hirschfield, P., Gasper, J. (2011). Relationship between school engagement and delinquency in late childhood and early adolescence. Journal of Youth and Adolescence, 40, 3-22.

Janosz, M., Archambault, I., Morizot, J., \& Pagani, L. (2009) Adolescent behavioral, affective, and cognitive engagement in school: Relationship to dropout. Journal of School Health, 79(9), 408-415. doi: 10.1111/j.1540-4560.2008.00546.

Jessor, R., \& Jessor, S. L. (1977). Problem behavior and psychosocial development: A longitudinal study of youth. New York, NY: Academic Press.

Kenney-Benson, G. A., Pomerantz, E. M., Ryan, A. M., \& Patrick, H. (2006). Sex differences in math performance: The role of children's approach to schoolwork. Developmental Psychology, 42, 11-26.

Kindermann, T. A. (2007). Effects of naturallyexisting peer groups on changes in academic engagement in a cohort of sixth graders. Child Development, 78, 1186-1203.
Kiuru, N., Nurmi J., Aunola K., Salmela, \& Aro, K. (2009). Peer group homogeneity in adolescents'school adjustment varies according to peer group type and gender. International Journal of Behavioral Development, 33 (1) 65-76.

Lansford, J. E. (2006). Peer effects in community programs. Dans K. A. Dodge, T. J. Dishion, \& J. E. Lansford (dir), Deviant peer influences in programs for youth: Problems and solutions (pp. 215233). New York, NY: Guilford.

Lam, S-F., Jimerson, S., Kikas, E., Cefai, C., Veiga, F.H., Nelson, B., Hatzichristou, C., \& al., (2012). Do girls and boys perceive themselves as equally engaged in school? The results of an international study from 12 countries. Journal of School Psychology, 50, 77-94.

Lanctôt, N. (2006). Un modèle théorique pour expliquer les conduites déviantes des adolescentes. Dans P. Verlaan et M. Déry (dir.). Les conduites antisociales chez les filles: Comprendre pour mieux agir (p. 121-147). Québec, QC : Presses de l'Université du Québec.

Lanctôt, N. (2005). Lien entre l'inadaptation scolaire des adolescents et des adolescentes judiciarisés et leur adaptation sociale et personnelle à l'âge adulte. Nouveaux Cahiers de la Recherche en Éducation, 8(2), 89-100.

Lanctôt, N., Leblanc, M. (1997). Les adolescentes de bandes marginales : un potentiel antisocial atténué par la dynamique de bande ? Criminologie, 30(1), 111-130.

Larson, R. W. (2000). Toward a psychology of positive youth development. American Psychologist, 55, 170-183. doi: 10.1037/0003-066X.55.1.170

Loeber, R., Farrington, D.P. StouthamerLoeber, M., \& Van Kammen, W. B. (1998). Antisocial behavior and mental health problems: Explanatory factors in childhood and adolescence. Mawhaw, $\mathrm{NJ}$ : Lawrence Erlbaum.

Lott, B. E. (1961). Group Cohesiveness: A learning phenomenon. Journal of Social Psychology, 55, 275-286. 
Mahoney, J. L., \& Stattin, H. (2000). Leisure activities and adolescent antisocial behavior: The role of structured and social context. Journal of Adolescence, 23, 113-127.

Marcus, R. F. (1996). The friendships of delinquents. Adolescence, 31, 145-158.

Marsh, H. (1992). Extracurricular activities: Beneficial extension of the traditional curriculum or subversion of academic goals? Journal of Educational Psychology, 84, 553-562.

Marsh, H.W., \& Kleitman, S. (2002) Extracurricular school activities: The good, the bad and the nonlinear. Havard Educational Review, 72, 464-493.

McCarthy, B., Felmlee, D., \& Hagan J. (2004). Girl friends are better: Gender, friends, and crime among school and street youth. Criminology, 42, 805-835.

McHale, S. M., Crouter, A. C., \& Tucker, C. J. (2001). Free-time activities in middle childhood: Links with adjustment in early adolescence. Child Development, 72, 1764-1778.

Moffitt, T. E. (1993). Adolescence-limited and life-course-persistent antisocial behavior: A developmental taxonomy. Psychological Review, 100, 674-701.

Moretti, M. M., Odgers, C. L., \& Jackson, M. A. (2004). Girls and aggression: Contributing factor and intervention principles. New York, NY: Kluwer.

Morizot, J., \& Le Blanc, M. (2000). Le rôle des pairs dans l'émergence et le développement de la conduite délinquante: une recension critique des écrits. Revue de Psychoéducation, 29, 87-117.

Mortimer, J. T., Vuolo, M., Staff, J., Wakefield, S., \& Xie, W. (2008). Tracing the timing of career acquisition in a contemporary youth cohort. Work and Occupations, $35,44-84$.

Mounts, N. S., and Steinberg, L. (1995). An Ecological Analysis of Peer Influence on adolescent grade point average and drug use. Developmental Psychology, 31(6), 915-922. doi:10.1037/0012-1649. 31.6.915
Mucchielli, L. (1999). La déviance : normes, transgression et stigmatisation. Sciences Humaines, 99, 20-25.

Newcomb, M. D., Abbott, R. D., Catalano, R. F., Hawkins, J. D., Battin-Pearson, S., \& Hill, K. (2002). Mediational and deviance theories of late high school failure: Process roles of structural strains, academic competence, and general versus specific problem behavior. Journal of Counseling Psychology, 49 (2), 172-186.

Newmann, F. M. (1992). Student engagement and achievement in American secondary schools. New York, NY: Teachers College Press.

Osgood, D. W., Wilson, J. K., O'Malley, P. M., Bachmman, J. G., \& Johnson, L. D. (1996). Routine activities and individual deviant behavior. American Sociological Review, 61, 635-655.doi: 10.2307/2096397

Pellegrini, A. D., \& Blatchford, P. (2000). The child at school: Interactions with peers and teachers. New York, NY: Oxford University Press.

Roché, S. (2001). La délinquance des jeunes. Le Seuil, Paris.

Rubin, K. H., Bukowski, W., Parker, J., \& Bowker, J. C. (2008). Peer interactions, relationships, and groups. Dans W. Damon et R. Lerner (dir.), Developmental Psychology: An Advanced Course (pp.141-180). New York, NY: Wiley.

Rumberger, R. W. (1987). High school dropouts:Areview ofissues and evidence. Review of Educational Research, 57, 101-121. doi: $10.2307 / 1170232$

Rumberger, R. W. et Larson, K. A. (1998). Student mobility and the increased risk of high school dropout. American Journal of Education, 107, 1-35.

Ryan, A. M., \& Patrick, H. (2001). The classroom social environment and changes in adolescents' motivation and engagement during middle school. American Educational Research Journal, 38, 437460. doi: $10.3102 / 00028312038002437$ 
Sage, N. A., \& Kindermann, T. (1999). Peer networks, behavior contingencies, and children's engagement in the classroom. Merrill-Palmer Quarterly, 45, 143-171.

Sirin, S. R., Rogers-Sirin, L. et Collins, B. (2010). A measure of cultural competence as an ethical responsibility: Quick racial and ethical sensitivity test (Q-REST). Journal of Moral Education, 39(1), 49-64.

Stattin, H., Kerr, M., Mahoney, J. L., Persson, A., \& Magnusson, D. (2005). Explaining why a leisure context is bad for some girls and not for others. Dans J. L. Mahoney, R. Larson, \& J. S. Eccles (dir.), Organized activities as contexts of development: Extracurricular activities, after-school and community programs (pp. 211-234). Mahwah, NJ: Erlbaum.

Stanard, P., Belgrave, F. Z., Corneille, M. A., Wilson, K. D., \& Owens, K. (2010). Promoting academic achievement: the role of peers and family in the academic engagement of African American adolescents. Journal of Prevention and Intervention in the Community, 38(3), 198-212. doi: $10.1080 / 10852352.2010 .486298$

Strambler, M. J., \& Weinstein, R. S. (2010). Psychological disengagement in elementary school among ethnic minority students. Journal of Applied Developmental Psychology, 31(2), 155165. doi: http://dx.doi.org/10.1016/j. appdev.2009.11.006

Turner, R. J. et Avison, W. R. (2003) Status variations in stress exposure: Implications for the interpretation of research on race, socioeconomic status, and gender. Journal of Health and Social Behavior, 44(4), 488-505.

Vaillancourt, T., \& Hymel, S. (2006). Aggression, social status and the moderating role of sex and peer-valued characteristics. Aggressive Behavior, 32, 396-408.
Verlaan, P., \& Déry, M. (2006). Les conduites antisociales des filles. Québec, Qc. Presses de I'Université du Québec.

Veronneau, M-H., \& Dishion, T. J. (2011). Middle school friendships and academic achievement in early adolescence: A longitudinal analysis. Journal Early Adolescence, 31(1), 99-124.

Warrington, M., Younger, M. et Williams, J. (2000) Student attitudes, image and the gender gap. British Educational Research Journal, 26(3), 393-407.

Wiesner, M., Vondracek, F. W., Capaldi, D. M., \& Porfeli, E. (2003). Childhood and adolescent predictors of early adult career pathways. Journal of Vocational Behavior, 63, 305-328.doi: 10.1016/ S0001-8791(03)00028-9.

Youniss, J., McLellan, J. A., Su, Y., Yates, M. (1999). The role of community service in identity development: Normative, unconventional, and deviant orientations. Journal of Adolescent Research, 14, 249-262.

Zepke, N., \& Leach, L. (2010). Improving student engagement: Ten proposals for action. Active Learning in Higher Education, 11(3), 1-27.

Zill, N., Nord, C. W., \& Loomis, L. S. (1995). Adolescent time use, risky behavior, and outcomes: An analysis of national data. Rockville, MD, Westat, Inc..

Zimmerman, B. J., Martinez-Pons, M. (1990). Student differences in self-regulated learning: Relating grade, sex, and giftedness to self-efficacy and strategy use. Journal of Educational Psychology, 82, 51-59. 